\title{
Analyzing the Effect of Preprocessor Annotations on Code Clones
}

\author{
Sandro Schulze \\ University of Magdeburg \\ sanschul@ovgu.de
}

\author{
Elmar Jürgens \\ Technische Universität München \\ juergens@in.tum.de
}

\author{
Janet Feigenspan \\ University of Magdeburg \\ feigensp@ovgu.de
}

\begin{abstract}
The $C$ preprocessor cpp is a powerful and language-independent tool, widely used to implement variable software in different programming languages $(C, C++)$ using conditional compilation. Preprocessor annotations can used on different levels of granularity such as functions or statements. In this paper, we investigate whether there is a relation between code clones and preprocessor annotations. Specifically, we address the question whether the discipline of annotation has an effect on code clones. To this end, we perform a case study on fifteen different $C$ programs and analyze them regarding code clones and \#ifdef occurrences. We found only minor effects of annotations on code clones, but a relationship between annotations that align with the code structure (and code clones). With this work, we provide new insights why code clones occur in $C$ programs. Furthermore, the results can support the decision whether or not it is beneficial to remove clones.
\end{abstract}

\section{Introduction}

The cpp preprocessor is a powerful text processing tool tightly coupled with the $\mathrm{C}$ programming language [1]. Due to its token-based nature, the cpp is language-independent and provides easy mechanisms to express variable source code using conditional compilation. In fact, preprocessor directives (or annotations) ${ }^{1}$ such as \#ifdef or \#ifndef can be used on any level of granularity.

1. Although the cpp tool supports different kinds of preprocessor directives, such as file inclusion or macro definition, we focus only on conditional inclusion within this paper.
Conversely, this flexibility makes it a root for poor code quality, caused by the missing structure of the cpp tool. Amongst others, the cpp is considered to be error prone and to impair readability and maintainability of the code [2], [3], [4], [5]. A pivotal role for the effect of annotations on source-code quality is whether these annotations are disciplined or undisciplined. It is commonly accepted that undisciplined annotations contribute to unstructured, tangled source code with the mentioned negative effects [6], [2], [7], [8].

In Figure 1, we show two code fragments containing conditional code that is undisciplined and disciplined, respectively. Undisciplined annotations (Figure 1(a), Line 3-5 and 8-10) are made on arbitrary syntactical units, such as parameters or branch conditions, and do not align with the overall code structure. By contrast, disciplined annotations (Figure 1(b)) are mapped to corresponding syntactical units such as functions or statements and thus align with the code structure. As a result, it is commonly accepted that disciplined annotations alleviate the drawbacks of annotations on sourcecode quality [2], [9], [10]. However, we and others observed that disciplined annotations may lead to replicated code fragments, commonly known as code clones [6].

Code clones are common in software development [11], [12], [13]. Furthermore, several studies reveal that code clones have a negative effect on software structure, leading to increased maintenance effort, inconsistent changes, and introduction of errors [14], [15], [16], [17]. Beyond that, several studies exist that aim at identifying causes for code clones as well as evaluating the effect and occurrence of clones on the software system [18], [19], 


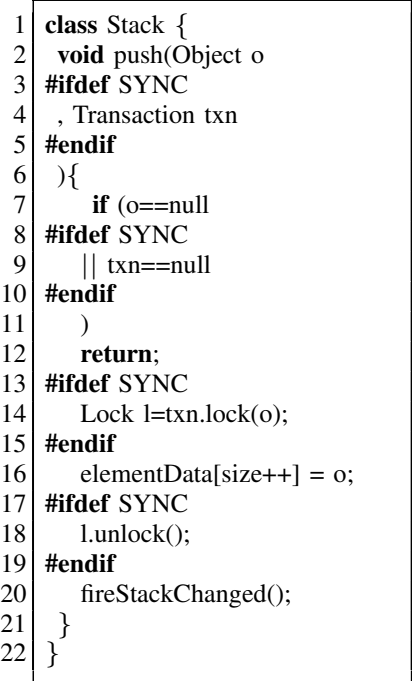

(a) undisciplined

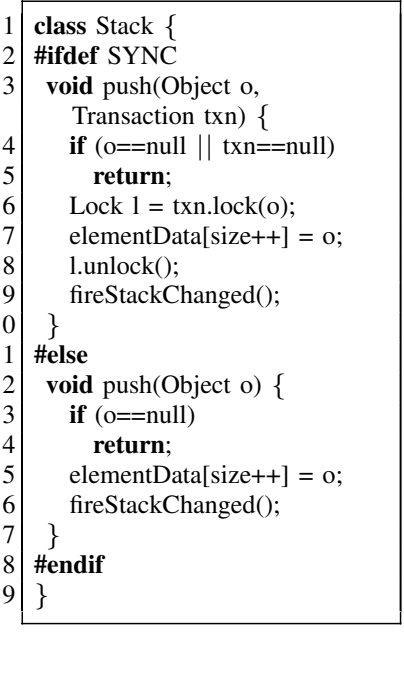

(b) disciplined
Figure 1. Two Examples for undisciplined and disciplined annotation usage.

[20]. However, these studies do not investigate the effect of preprocessor annotations.

Research problem. Disciplined annotations align with the source code and thus limit expressiveness. On the other hand, they alleviate the drawbacks of annotations on source code quality. Furthermore, recent observations indicate that such annotations cause code clones. However, up to now it is unclear whether disciplined annotations lead to an increased amount of code clones in practice. If this is the case, this poses the question whether replacing undisciplined annotations by disciplined ones (at the expense of code clones) really improve code quality.

Contribution. In this work, we extend existing studies by a large case study that investigates the relation between code clones and preprocessor annotations. As a result, we provide new insights on why code clones occur. Furthermore, the information of our analysis can be useful to support the decision whether to remove clones or not.

\section{Background}

A variety of research has been done for both code clones and preprocessor directives. In this section, we give an overview of terms and definitions relevant to this paper.

\subsection{Code Cloning}

Code clones are source code fragments that are similar to each other. Different types of clones exist depending on the degree of similarity between two code fragments [21]. Code fragments that are identical are called type-I or exact clones. Furthermore, code clones that differ only slightly are called typeII clones. For instance, differences due to renaming of variables or constants typically lead to typeII clones. Finally, type-III or gapped clones are similar code fragments that differ due to adding, removing, or changing code units of at least one of the code fragments. Finally, two code fragments that are identified as code clones are called a clone pair. Additionally, a set that consists of two or more code clones is a clone group.

Different approaches exist to detect the different types of code clones. First, text-based approaches use simple string or character comparison and thus detect primarily type-I clones [22]. Second, tokenbased approaches perform a tokenization on the source code and compare these tokens to detect type-I and type-II clones [23]. Third, AST-based approaches create a grammar-based abstraction of the source code, the Abstract Syntax Tree (AST). A sophisticated variant is the $P D G$-based approach that additionally takes data and program flow into account. Both approaches detect type-I and typeII clones, based on their internal source code representation [12], [24]. Finally, some approaches can even detect type-III clones such as ConQAT ${ }^{2}$, which defines a threshold for the maximum edit distance between clones [25]. For a comprehensive overview of clone detection techniques we refer to Roy et al. [26].

2. http://www.conqat.org 


\subsection{Annotations in cpp}

Annotations, or more specifically conditional inclusion using \#ifde $f^{3}$, can be used to generate different variants (with different functionality) of a program [7]. Each annotation contains a boolean expression that is evaluated by the cpp tool to determine whether the conditional code is included in a certain program or not. Usually, such an expression represents a feature, an increment in user-visible functionality [27].

In general, annotations can be classified into two categories, based on the syntactical units they annotate: disciplined and undisciplined annotations [6]. This, in turn, raises the question where the borderline between these two categories is. Obviously, defining such a borderline depends on several criteria. Liebig et al. propose a definition for disciplined annotations, according to which annotations of one or a sequence of functions, type definitions, statements, and elements inside type definitions are disciplined [6]. In contrast, annotations such as on function parameters are undisciplined based on this definition. We rely on this definition within this paper because it is reasonable and suitable for our purposes. For clarification, we depict some examples for both kinds of annotations in Figure 2. For more details, please refer to [6].

\section{Code Clone Analysis Process}

In this section, we describe the connection between cpp usage and code clones, infer two research questions and explain the design of our case study.

\subsection{Research Questions}

We perform the case study to gain insights on the relation between code clones and annotations in preprocessor-based programs. To this end, we formulate the following research questions:

3. Within this paper, \#ifdef is a placeholder for all possibilities of conditional inclusion: \#ifndef, \#if, \#elif, \#else and \#endif

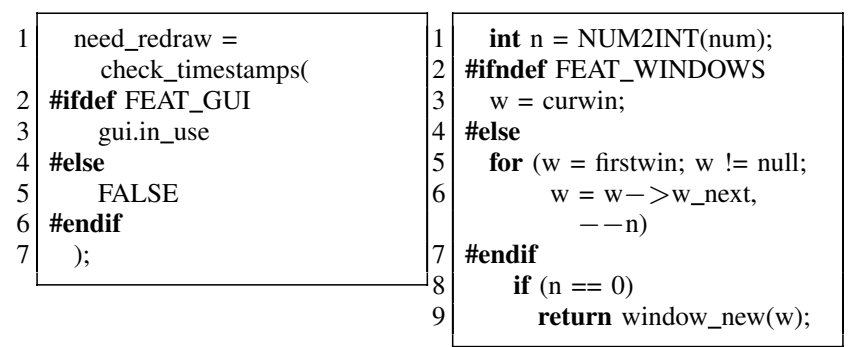

(a) examples of undisciplined annotations

\begin{tabular}{l|l|}
\cline { 2 - 3 } & void tcl_end() \{ \\
2 & \#ifdef DYNAMIC_TCL \\
3 & if (hTclLib) \{ \\
4 & FreeLibrary(hTclLib); \\
5 & hTclLib = NULL; \\
6 & \} \\
7 & \#endif \\
8 & T
\end{tabular}

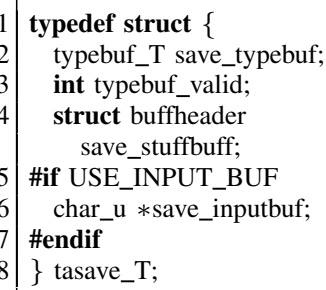

(b) examples of disciplined annotations

Figure 2. Examples for undisciplined and disciplined annotation

RQ 1 To what extent do code clones occur in annotated \#ifdef blocks?

Several studies reveal the existence of code clones in $\mathrm{C}$ programs. However, none of these studies analyzes how much of the detected code clones occur in preprocessor blocks. We aim at answering this question to better understand to what extent preprocessors cause code clones, independent of their granularity.

RQ 2 Are there differences between disciplined and undisciplined annotations regarding code clone occurrence?

This question is motivated by the observation that disciplined annotations may come at the expense of introducing code clones [6]. Consequently, we evaluate whether this observation is accidental or may depend on the discipline of annotations. Answering this question may also affect the evaluation of code clones regarding their harmfulness. For instance, if a code clone is introduced in order to overcome undisciplined annotations, should this clone considered harmful? As a direct consequence, this information may also support refactoring decisions in terms of code clone removal. 


\subsection{Study Design}

We perform a code clone analysis supplemented by clone detection and source code analysis. The detailed process is described in Section 3.3. As a result, we collect information on the amount of code clones, \#ifdef code (i.e., code that is contained between \#ifdefs), and \#ifdef clones (i.e., code clones that are enclosed by \#ifdefs). Then, we compute different measures based on these results.

First, we compute the code clone and \#ifdef coverage. The term coverage denotes the part of the source code that is covered by code clones or \#ifdef blocks. These measures provide us with general information on the systems and whether it is worth to investigate these systems further.

Second, we compute the \#ifdef clone coverage to investigate how much of the overall code contains \#ifdef clones. Additionally, we compute the ratio of \#ifdef clones compared to a) all detected code clones (\#ifdef-clone/clone ratio) and b) the total amount of annotated code (\#ifdef-clone/\#ifdef ratio). With these measures, we can determine whether there are other correlations that are likely to cause \#ifdef clones.

Finally, we compute all of our measures for disciplined and undisciplined systems (cf. Section 3.4) separately and thus, can compare both categories.

\subsection{Analysis of \#ifdef Clones}

In this section, we give an overview of the design of our code clone analysis process. For answering our research questions, we set up a three-staged process, which we depict in Figure 3. In the following we explain the three phases clone detection, source code analysis, and code clone analysis in detail.

Clone Detection. For clone detection, we use ConQAT, a token-based clone detection tool that can detect gapped clones. We chose to detect type3 clones, because they may occur within disciplined annotations, as indicated by our example in Figure 1. Initially, the source code is transformed into a token sequence from which comments and whitespaces are removed. Afterwards, ConQAT

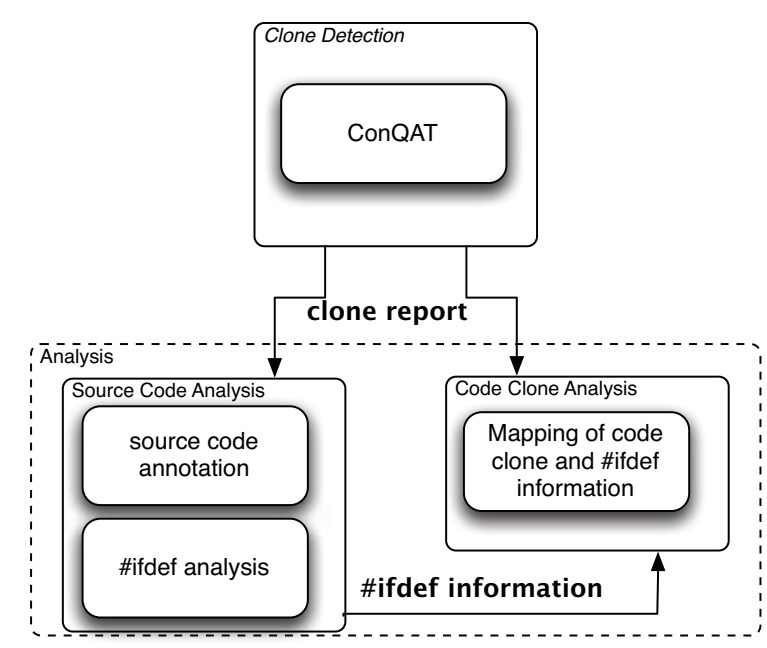

Figure 3. Outline of the clone analysis process

performs normalization on the token sequence, which can be divided into two parts. First, statements are created from the token sequence since it leads to better clone detection results (e.g., ignoring clones that start/end within statements). Second, tokens are normalized by user-defined rules, which eliminates differences between the specified syntactical units such as identifiers or constants. For instance, we set up the normalization in such a way that differences between literals values of the same type (e.g. boolean or int) are ignored for the actual clone detection. However, we do not normalize differences between identifiers to preserve high precision.

Finally, the clone detection is performed on the normalized token sequence. In a nutshell, a suffix tree is built on the token sequence and then, the algorithm searches for all identical or similar substrings in the tree. The user can influence the clone detection result by specifying different parameters such as the minimum clone length. For our purposes, we selected a minimum clone length of eight statements. Furthermore, we performed a gapped clone detection, so that gapped clones are detected as well. Therefore, we have to specify the gap ratio, a measure that determines the maximum number of gaps between two code clones. We selected a gap ratio of 0.25 , which means for a 
clone pair of eight statements that two statements of at least one clone may have been deleted, added, or changed.

The result of the clone detection is subject to post-processing such as filtering out selfoverlapping clone groups. Furthermore, remove clones that we detect in generated code. Finally, a clone report is generated, containing information on all source files as well as on all clone groups and its corresponding code clones, which can be used for further usage. For a more detailed description of the clone detection algorithm, we refer to [14].

Source Code Analysis. For obtaining information on occurrences of annotations, we have to analyze the source code that was subject to clone detection in the previous step. To this end, we annotate corresponding source files using $\operatorname{src} 2 \mathrm{srcml}^{4}$, a source code markup language that annotates the source code in an xml-like fashion without breaking its overall structure [28]. Afterwards, we detect \#ifdefs in the annotated code using XPath, an xml path language that can be used to navigate through the nodes of an xml document. As a result, we obtain all occurrences of \#ifdef annotations, identified by their absolute position (i.e., line number) in the source file. Note, that we get this information for complete \#ifdef blocks, that is, code fragments that are enclosed by annotations such as \#ifdef and \#endif. Finally, the results of this analysis can be used for code clone analysis.

Code Clone Analysis. For our analysis, we map the detected code clones to the detected \#ifdef annotations, based on their absolute position in the source file. We illustrate the mapping algorithm in Figure 4.

Our algorithm has two inputs: A list of all clone groups from the clone report, and a list of preprocessor annotations together with the information where they can be found (i.e., the file and the line number). For the mapping, we consider the code clones of each clone group separately. Then, we compare the position of each clone with the positions of all preprocessor annotations that have been found in the file containing the clone. We have

\footnotetext{
4. http://www.sdml.info/projects/srcml/
}

\author{
proc mapClones $(c g, p a)$ \\ Input: $\mathrm{cg}=$ list of clone groups, $\mathrm{pa}=$ list of \\ annotations \\ for each $c g_{i} \in c g$ do \\ get all code clones from $c g_{i}$ \\ for each clone do \\ $f a \subset p a=$ all annotations for the file \\ containing the clone \\ if $(a \in f a)$ is within clone then \\ clone $^{\prime}=$ new clone with the position of \\ a \\ create new ifdef clone group with clone' \\ (if this is the first clone of $c g_{i}$ ) \\ otherwise add clone $e^{\prime}$ to existing ifdef \\ clone group (for $c g_{i}$ ) \\ end if \\ end for \\ end for \\ Result: list of \#ifdef clone groups
}

Figure 4. Algorithm for mapping annotations to code clones.

a match, if at least one complete \#ifdef block is within the clone. Hence, \#ifdef blocks that are not entirely located within a code clone (e.g., they start or end outside the clone) are ignored. We imposed this restriction because incomplete clones such as statements at the end of a conditional branch pose a high risk to be meaningless or even incidental. As a consequence, this may lead to less accurate results.

Furthermore, the \#ifdef block must have a length of at least five source lines of code, including the lines containing the annotations. This is threshold has been used by others [29] and worked well for us, too. In case of a match, we create a new \#ifdef clone. We do this for all clones of a clone group. Finally, all corresponding ifdef clones are merged to an \#ifdef clone group.

\subsection{Study Objects}

For our case study, we use fifteen software systems written in the $\mathrm{C}$ programming language. We selected programs of different size and domains 
to have a representative sample. Furthermore, to evaluate our second research question, we split the sample in two comparable groups: Seven systems are disciplined (i.e., contain almost no undisciplined annotations), eight systems undisciplined (i.e., contain $12 \%$ undisciplined annotations on average). The classification is based on a recent study of Liebig et al., who analyzed the discipline of annotations in C programs [6]. Beyond that, both groups are comparable regarding size and domains. In Table 1, we give an overview of the analyzed programs.

\begin{tabular}{|c|c|c|c|}
\hline & program & \# SLOC & description \\
\hline \multirow{8}{*}{ 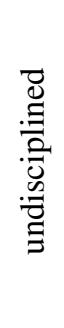 } & cherokee & 47983 & Web server \\
\hline & gnuplot & 67854 & plotting tool \\
\hline & $\operatorname{lyn} x$ & 111994 & Web browser \\
\hline & php & 471604 & program interpreter \\
\hline & privoxy & 24784 & proxy server \\
\hline & sendmail & 85094 & mail transfer agent \\
\hline & tcl & 122460 & program interpreter \\
\hline & $\operatorname{vim}$ & 233426 & text editor \\
\hline \multirow{7}{*}{ 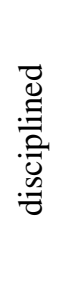 } & berkeleyDB & 160283 & database system \\
\hline & dia & 121117 & diagramming software \\
\hline & ghostscript & 491703 & postscript interpreter \\
\hline & lighttpd & 37380 & Web server \\
\hline & $\operatorname{minix}$ & 54627 & operating system \\
\hline & parrot & 84222 & virtual machine \\
\hline & python & 331014 & program interpreter \\
\hline
\end{tabular}

Table 1. Overview of analyzed C Programs.

\section{Results}

In this section, we present case study results.

First of all, we observed that in all systems, annotated code as well as code clones exist. We show the results in Figure 5. In undisciplined systems (Figure 5 a) the coverage of annotated code is $32,7 \%$, whereas it is $16,7 \%$ on average for the disciplined systems (Figure $5 \mathrm{~b}$ ). The disciplined systems exhibit a higher clone coverage (10\% on average, $8,7 \%$ on median) compared to the undisciplined systems $(4,3 \%$ on average, $2 \%$ on median). Furthermore, six of eight undisciplined systems have a clone coverage less than $3 \%$. Nevertheless, all systems contain code clones as
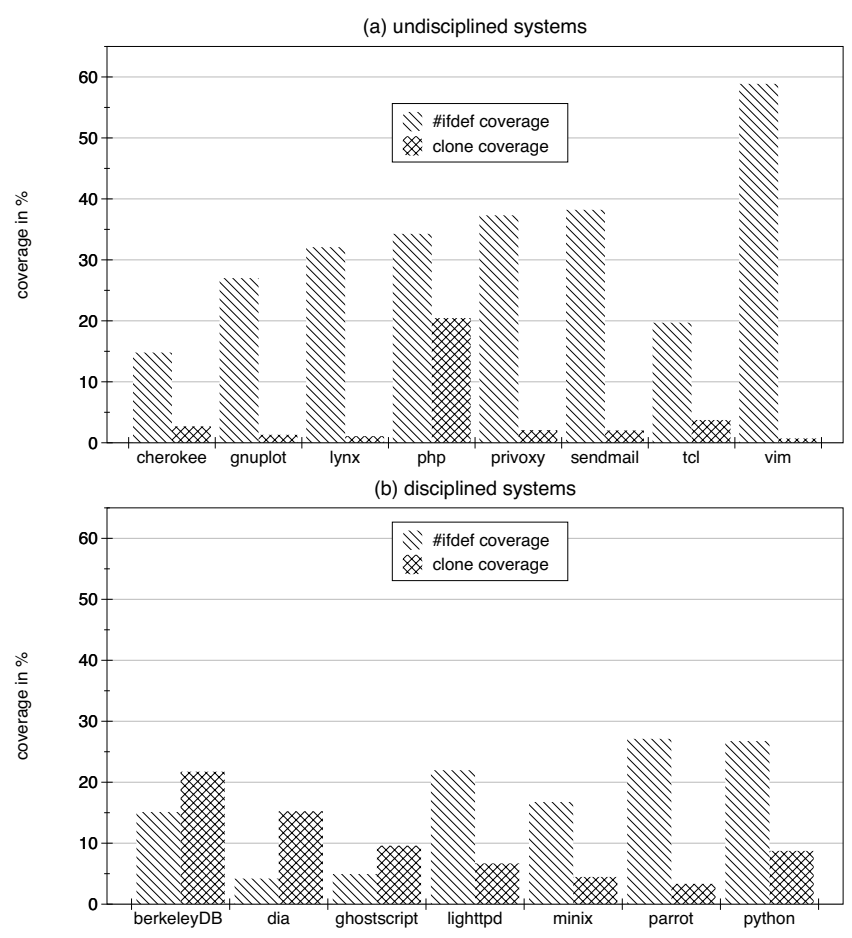

Figure 5. Clone and \#ifdef coverage of the analyzed systems

well as \#ifdef annotated code in a reasonable amount and thus, are further investigated.

To evaluate RQ 1, we measured the \#ifdef clone coverage supplemented by the clone/\#ifdef-clone ratio and the \#ifdef/\#ifdef-clone ratio (see Section 3.2 for definition). We depict our results in Figure 6. The scatter plot in Figure $6 \mathrm{a}$ indicates that the \#ifdef clone coverage is rather small. Indeed, only four systems exhibit a coverage of more than $0,5 \%$, and only one system (BerkeleyDB) has an \#ifdef clone coverage higher than $2 \%$. In the same way, Figure $6 \mathrm{~b}$ indicates that only a minor fraction of all detected code clones occur within \#ifdef blocks, independent of the actual amount of clones. Similarly, only a small fraction of the overall \#ifdef code contains code clones (Figure 6c). We observed that those systems with the highest amount of \#ifdef code contain only few \#ifdef clones. By contrast, amongst the systems with a rather small amount of \#ifdef code $(<25 \mathrm{~K}$ SLOC), in particular systems \#ifdef code contains up to $14 \%$ \#ifdef clones. 

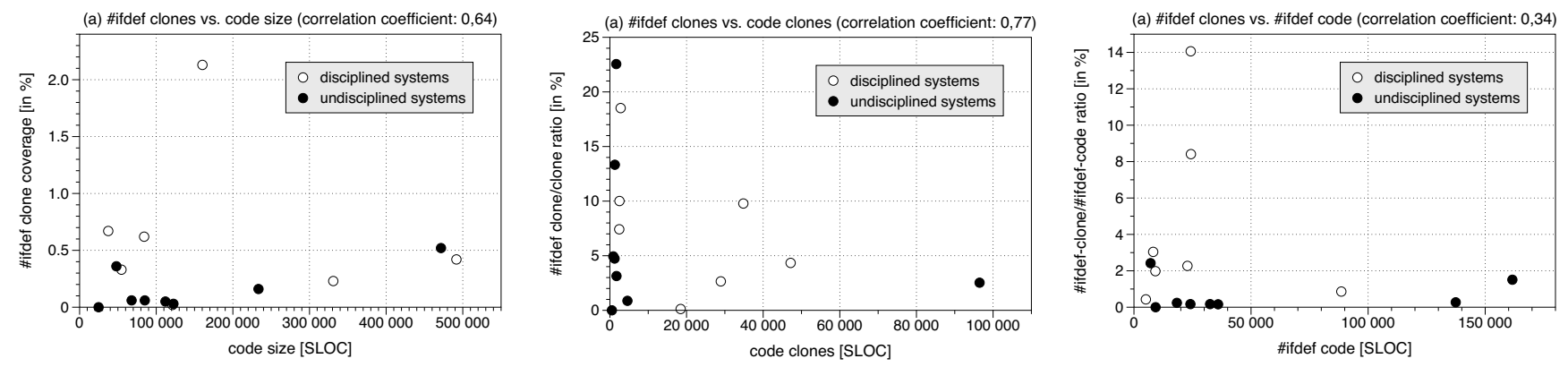

Figure 6. Analysis results for \#ifdef clones: a) w.r.t the overall code size, b) w.r.t all code clones c) w.r.t annotated code

With RQ 2, we aim at investigating whether there are differences between disciplined and undisciplined systems regarding the amount of \#ifdef clones. Therefore, we compare the amount of \#ifdef clones in disciplined and undisciplined systems. Although \#ifdef clone coverage is rather low, Figure 6 a reveals that the amount of \#ifdef clones is considerably higher in disciplined systems. We observed that five of the eight undisciplined systems have a coverage close to zero and the average \#ifdef clone coverage is $0,15 \%$. By contrast, four of the seven disciplined systems have an \#ifdef clone coverage of approximately $0,5 \%$ or higher $(0,63 \%$ in average). Furthermore, we observed that annotated code in disciplined systems is more likely to contain clones than in undisciplined systems (cf. Figure 6c). Although the latter systems contain more annotated code, in six systems this code contains nearly no code clones. Contrary, out of the six system that contain $2 \%$ or more code clones in \#ifdef blocks, five are disciplined systems. All of these observations are confirmed by a medium or even high correlation coefficient we computed, using the method of Pearson.

\section{Discussion}

In the following we interpret the results we presented in the previous section and discuss threats to validity of our case study.

\subsection{Interpretation of Results}

The results of RQ 1 reveal that the amount of code clones in preprocessor annotation is rather small (with minor exceptions). This especially holds for the systems with a high amount of undisciplined annotations, where all measured data indicate that \#ifdef clones are negligible. However, due to our very strict definition of \#ifdef clones (only complete annotated blocks are considered), this result can be interpreted as a lower bound. Consequently, a more fine-grained investigation of \#ifdef clones, where partial \#ifdef clones are allowed up to a certain threshold, may even increase their overall amount. Beside this, a larger case study with more systems can also support the investigation of \#ifdef clones in cpp-based programs.

For RQ 2, the results indicate that \#ifdef clone coverage is significantly higher for disciplined systems compared to undisciplined systems. This, in turn, confirms the assumption that disciplined annotations lead to code clones. Furthermore, our data reveal that other factors such as code size and amount of code clones do not influence this observation and thus can be neglected.

To evaluate whether the observed differences between disciplined and undisciplined systems are significant or rather occurred randomly, we conducted a significance test. We applied an adapted version of the Mann-Whitney-U test: Instead of providing the significance level of the test, we check whether the calculated $U$ values are signifi- 
cant according to a table specifically designed for small sample sizes [30], [31]. The results of the Mann-Whitney-U test reveal that the differences for the \#ifdef/\#ifdef-clone ratio as well as the \#ifdef clone coverage are significant. First, for the \#ifdef/\#ifdef-clone ratio, we obtained the following results: $\mathrm{U}=6, \mathrm{p}<0.01$. Second, for the \#ifdef clone coverage our test produced the following results: $U=12, p<0.05$. Since both significance levels are smaller than 0.05 , we can assume that the differences we observed are significant and not caused randomly.

Revisiting the introduced research problem, our study results indicate that disciplined annotations increase the amount of code clones compared to undisciplined one. However, due to the small \#ifdef clone coverage, the effects of these clones may be not as negative as for undisciplined annotations. Consequently, from our point of view, the benefits of disciplined annotations such as tool support [6] outweigh the drawbacks of code cloning, especially considering the low absolute amount of clones in annotated code.

\subsection{Threats to Validity}

Single Programming Language. Although the cpp tool is language-independent and thus used with several programming languages, we only considered C programs within our study for three reasons. First, for the selected systems we had prior knowledge about the discipline of annotations, based on the study of Liebig et al. [6]. Second, with the decision for one specific language, we can prevent that certain mechanisms of different languages influence the amount of (\#ifdef) code clones. Finally, the cpp tool has been used with $\mathrm{C}$ programs for a long time and thus the systems are mature and different case studies exist. Overall, we assume that this decision may limit generalizability but does not affect our findings.

Selected Software Systems. With case studies on software systems, there is a risk that the selected systems bias the study results. To mitigate this effect, we selected systems of different size and of different domains as far as this was possible.
Clone Detection. Both initial clone detection as well as \#ifdef clone detection have been performed automatically, based on certain input parameters. Due to the large code amount, it is infeasible to check each clone regarding the precision of the clone detection. However, we randomly selected samples (for code clones and \#ifdef clones) from each subject system for a manual review process. All of these samples were true code clones that is, we detected no false positives. Furthermore, for the clone detection we selected parameters that are commonly accepted to avoid that meaningless or even false code clones are detected.

Study evaluation. During interpreting the results of our case study, we made several observations regarding our research questions. To strengthen our results, we conducted statistical computations, namely Pearson's correlation coefficient and a significance test. The main problem of the statistical evaluation is the quite small sample set represented by the fifteen subject systems. Although the statistic results indicate the correctness of the relations we observed, we have to be careful with the conclusions we draw. Hence, a larger case study with more systems is necessary. Nevertheless, our study results provide first insights on the relation between preprocessor annotations and code clones.

\section{Related Work}

For both, the preprocessor cpp as well as code cloning, we give an overview of prior work that is related to ours.

For cpp, Ernst et al. conducted a large case study that investigates the usage of preprocessor annotations and its implications [2]. In their work, they highlight advantages of disciplined preprocessor use and why this fails regularly. However, they mainly focus on usage of macro definition such as \#define and thus conditional inclusion is just mentioned partly. Recently, Liebig et al. presented comprehensive results regarding preprocessor usage on which we partially base our paper. Particularly, they analyzed preprocessor annotations in the context of software product lines by 
means of a large case study. First, they defined several metrics for measuring system properties such as granularity or types of extensions [32]. Second, they analyzed the discipline of annotations in cpp-based programs [6]. In this context, they give a definition for disciplined annotations and conducted a case study on how disciplined and undisciplined annotations are used. However, they neither considered the occurrence of code clones within their study nor the usage of certain \#ifdefs (e.g., disciplined ones) to avoid clones.

Amongst the several case studies on code clones, some of them specifically focus on clones in $\mathrm{C}$ programs. First, Mayrand et al. presented an experiment on function clones in C programs [18]. Within their work they propose a taxonomy for function clones as well as different notions of similarity, based on metrics. However, their code clone analysis focus only on function blocks and thus, they do not consider preprocessors as we do. Second, Roy et al. conducted a large case study on code clones in open source systems (C \& Java) [19]. They propose different metrics such as clone density, clone location and clone size for comprehensive insights on cloned code and verified their results manually. Nevertheless, they also focus rather on clones on function level. By contrast, we considered code clones in the context of preprocessor annotations.

\section{Conclusions and Future Work}

In this paper, we conducted an empirical study on fifteen $\mathrm{C}$ systems to analyze the effect of preprocessor annotations on code clones. We detected only minor relations between annotations and code clones. Hence, we conclude that preprocessor annotations may only have a minor effect on code cloning. Beyond that, we observed significant differences between systems with disciplined and undisciplined annotations. Our results indicate that systems with disciplined annotations are more prone to code clones than systems with undisciplined annotations. Hence, our study provides sound findings to verify the common belief that disciplined \#ifdefs are prone to code clones.
However, due to the small amount of \#ifdef clones we argue that it is probably more beneficial to manage these clones instead of remove them (and probably introduce undisciplined annotations). To evaluate this claim, further studies are necessary to evaluate the harmfulness of these clones.

Besides evaluating the harmfulness of \#ifdef clones, there are more questions that remain unanswered: for what types of annotations do code clones occur in particular? Is there an overall relation between code clones and variability in cppbased systems? How would our results change for different types of clones (e.g., only type-II clones or partial \#ifdef clones) and more subject systems? In future work, we will focus on these questions to gain more insights on the relation between code clones and preprocessor annotations. Beyond that, we aim at providing a bigger picture of clones in the context of systems with high variability such as software product lines.

\section{Acknowledgement}

The authors would like to thank Jörg Liebig for helpful comments on the final version of this paper.

\section{References}

[1] B. W. Kernighan, The C Programming Language, 2nd ed. Prentice Hall, 1988.

[2] M. Ernst, G. Badros, and D. Notkin, "An Empirical Analysis of C Preprocessor Use," IEEE Trans. Soft. Eng., vol. 28, no. 12, pp. 1146 - 1170, 2002.

[3] B. Stroustrup, The Design and Evolution of C++. New York, NY, USA: Addison-Wesley, 1995.

[4] J. Favre, "Understanding-In-The-Large," in Proc. Int. Workshop on Program Comprehension. IEEE CS, 1997, pp. 29-38.

[5] H. Spencer and G. Collyer, "\#ifdef Considered Harmful, or Portability Experience with C News," in Proc. USENIX Tech. Conf. USENIX Association Berkeley, 1992, pp. 185-197.

[6] J. Liebig, C. Kästner, and S. Apel, "Analyzing the Discipline of Preprocessor Annotations in 30 Millions Lines of C Code," in Proc. Int. Conf. on Aspect-Oriented Software Development. ACM Press, 2011, pp. 191-202.

[7] C. Kästner, S. Apel, and M. Kuhlemann, "Granularity in Software Product Lines," in Proc. Int. 
Conf. on Software Engineering. ACM Press, 2008, pp. 311-320.

[8] C. Kästner, S. Apel, S. Trujillo, M. Kuhlemann, and D. Batory, "Guaranteeing Syntactic Correctness for All Product Line Variants: A Language-Independent Approach," in Proc. Int. Conf. on Objects, Models, Components and Patterns. Springer-Verlag, 2009, pp. 175-194.

[9] C. Kästner and S. Apel, "Virtual Separation of Concerns - A Second Chance for Preprocessors," J. Obj. Tech. (JOT), vol. 8, no. 6, pp. 59-78, 2009.

[10] I. Baxter and M. Mehlich, "Preprocessor Conditional Removal by Simple Partial Evaluation," in Proc. Work. Conf. on Reverse Eng. IEEE CS, 2001, pp. 281-291.

[11] B. S. Baker, "On Finding Duplication and NearDuplication in Large Software Systems," in Proc. Work. Conf. on Reverse Eng. IEEE CS, 1995, pp. 86-95.

[12] I. Baxter, A. Yahin, L. Moura, M. Sant'Anna, and L. Bier, "Clone Detection Using Abstract Syntax Trees," in Proc. Int. Conf. on Software Maintenance. IEEE CS, 1998, pp. 368-377.

[13] L. Jiang, G. Misherghi, Z. Su, and S. Glondu, "DECKARD: Scalable and Accurate Tree-based Detection of Code Clones," in Proc. Int. Conf. on Software Engineering. IEEE CS, 2007, pp. 96105.

[14] E. Jürgens, F. Deissenboeck, B. Hummel, and S. Wagner, "Do Code Clones Matter?" in Proc. Int. Conf. on Software Engineering. IEEE CS, 2009, pp. 485-495.

[15] B. Laguë, D. Proulx, J. Mayrand, E. Merlo, and J. Hudepohl, "Assessing the Benefits of Incorporating Function Clone Detection in a Development Process," in Proc. Int. Conf. on Software Maintenance. IEEE CS, 1997, pp. 314-321.

[16] L. Aversano, L. Cerulo, and M. Di Penta, "How Clones are Maintained: An Empirical Study," in Proc. Eur. Conf. on Software Maintenance and Reengineering. IEEE CS, 2007, pp. 81-90.

[17] M. Kim, V. Sazawal, D. Notkin, and G. Murphy, "An Empirical Study of Code Clone Genealogies," in Proc. Eur. Soft. Eng. Conf./Int. Symp. on Foundations of Soft. Eng. ACM Press, 2005, pp. 187196.

[18] J. Mayrand, C. Leblanc, and E. Merlo, "Experiment on the Automatic Detection of Function Clones in a Software System Using Metrics," in Proc. Int. Conf. on Software Maintenance. IEEE CS, 1996, pp. 244-253.

[19] C. Roy and J. Cordy, "Near-miss Function Clones in Open Source Software: An Empirical Study," Journal of Software Maintenance, vol. 22, no. 3, pp. 165-189, 2010.

[20] H. Basit and S. Jarzabek, "A Data Mining Approach for Detecting Higher-Level Clones in Software," IEEE Trans. Soft. Eng., vol. 35, pp. 497514, 2009.

[21] C. Roy and J. Cordy, "A Survey on Software Clone Detection Research," School of Computing, Queen's University at Kingston, Tech. Rep. 2007451, 2007.

[22] S. Ducasse, M. Rieger, and S. Demeyer, "A Language Independent Approach for Detecting Duplicated Code," in Proc. Int. Conf. on Software Maintenance. IEEE CS, 1999, pp. 109-118.

[23] T. Kamiya, S. Kusumoto, and K. Inoue, "CCFinder: A Multilinguistic Token-Based Code Clone Detection System for Large Scale Source Code," IEEE Trans. Soft. Eng., vol. 28, no. 7, pp. 654-670, 2002.

[24] J. Krinke, "Identifying Similar Code with Program Dependence Graphs," in Proc. Work. Conf. on Reverse Eng. IEEE CS, 2001, pp. 301-309.

[25] E. Jürgens, F. Deissenboeck, and B. Hummel, "CloneDetective - A Workbench for Clone Detection Research," in Proc. Int. Conf. on Software Engineering. IEEE CS, 2009, pp. 603-606.

[26] C. Roy, J. Cordy, and R. Koschke, "Comparison and Evaluation of Code Clone Detection Techniques and Tools: A Qualitative Approach," Science of Computer Programming, vol. 74, no. 7, pp. $470-495,2009$.

[27] C. Prehofer, "Feature-Oriented Programming: A Fresh Look at Objects," in Proc. Eur. Conf. on Object-Oriented Programming. Springer-Verlag, 1997, pp. 419-443.

[28] J. Maletic, M. Collard, and A. Marcus, "Source Code Files as Structured Documents," in Proc. Int. Workshop on Program Comprehension. IEEE CS, 2002, pp. 289-292.

[29] M. Bruntink, A. van Deursen, R. van Engelen, and T. Tourwé, "On the Use of Clone Detection for Identifying Crosscutting Concern Code," IEEE Trans. Soft. Eng., vol. 31, pp. 804-818, 2005.

[30] T. Anderson and J. Finn, The New Statistical Analysis of Data. Springer-Verlag, 1996.

[31] L. Giventer, Statistical Analysis for Public Administration, 2nd ed. Jones and Bartlett Publishing, 2008.

[32] J. Liebig, S. Apel, C. Lengauer, C. Kästner, and M. Schulze, "An Analysis of the Variability in Forty Preprocessor-Based Software Product Lines," in Proc. Int. Conf. on Software Engineering. ACM Press, 2010, pp. 105-114. 